\title{
A GRADE approach hits the mark in the new CUA male urethral stricture guideline
}

\section{R. Christopher Doiron, MD, FRCSC}

Department of Urology, Queen's University, Kingston, ON, Canada

Cite as: Doiron RC. A GRADE approach hits the mark in the new CUA male urethral stricture guideline. Can Urol Assoc J 2020;14(10):317-8. http://dx.doi.org/10.5489/cuaj.6940

See related article on page 305

$\mathrm{T}$ he latest clinical practice guideline from the Canadian Urological Association (CUA), authored by a venerable cast of Canadian reconstructive urologists and guideline methodologists, is a guideline on male urethral stricture. ${ }^{1}$ It is published in this month's edition of CUAJ and was constructed using the Grading of Recommendations Assessment, Development and Evaluation (GRADE) adolopment approach. ${ }^{2}$ Central to the adolopment approach in GRADE guideline development is updating and improving existing guidelines in an attempt at reducing redundancy in a world of medical literature recapitulation. To this end, the systematic review of the American Urological Association (AUA)'s male urethral stricture guideline, ${ }^{3}$ which included studies published up to January 2015, was updated in the current guideline with studies published from January 2014 to October 2018. This resulted in the identification of several new citations for inclusion into their evidence-based recommendations.

The authors opted to focus their formal recommendations to three distinct clinical questions with wide-reaching relevance to the management of urethral strictures, while the document finishes with an exploratory riff on several challenging stricture scenarios they felt warranted discussion.

In the first question examined, "Should a suspected diagnosis of urethral stricture be investigated with a cystoscopy as the most accurate method of diagnosis?" the author's suggest cystoscopy rather than urethrography for the initial diagnosis, suggesting a retrograde urethrogram and reconstructive urologist referral for further staging, while suggesting against routine imaging with magnetic resonance for diagnosis. Note the terminology used here (and in all the guideline's recommendations) is "suggest," which by GRADE methodology denotes a "conditional recommendation" based on a "low certainty in evidence of effects," versus "recommends," which would be based on a "strong recommendation" and a "high certainty in the evidence of effects." With respect to the suggestion of cystoscopy, no new studies beyond those identified by the AUA's guideline were included in its derivation.

The two suggestions that follow are with regards to treatment of male urethral stricture. In the first, endoscopic management (dilation or direct vision internal urethrotomy [DVIU]) is suggested in the initial treatment of an undifferentiated, symptomatic stricture - again, a "conditional recommendation," based mostly on non-comparative, observational data. In fact, of the 28 studies analyzed in the generation of this suggestion, 22 of which were newly included compared to the AUA guideline, only six were comparative in design. Importantly, this suggestion should not be applied to those patients presenting with any degree of complexity, including penile urethral strictures and strictures $>2 \mathrm{~cm}$ in length, among other complicating presentations. ${ }^{1}$

The final formal suggestion from the authors suggests urethroplasty for a stricture having failed endoscopic management. The suggestion is based on 32 studies -21 newly added to the 11 from the AUA's guideline - and is a "conditional recommendation." Only one study of the lot was comparative in design, while the remaining data were derived from non-comparative, observational studies. A "special circumstances" section rounds out the guideline and while lacking formal recommendations, covers six challenging stricture scenarios from trauma stenoses to lichen sclerosus to radiation-induced urethral stenoses. It represents a concise review of the literature and provides helpful guidance for those urologists managing more advanced urethral stricture patients.

The GRADE approach improves on historical methods of guideline development by explicitly including statements with regards to patient values and preferences, as well as including equity and feasibility implications into development of guideline suggestions or recommendations. ${ }^{4}$ This framework recognizes there is more to a clinical decision than just the magnitude of effect reported in the literature. When it comes to handling the data, the methodology moves away from the rigidity of a hierarchy-of-evidence model, incorporating data in a more balanced and nuanced manner. 
It is a rigorous process and is resource-intensive, but results in an evidence-to-decision framework that is good not just for the clinicians trying to navigate patient management in evidence-poor clinical scenarios, but also good for patients and society, given the inclusive nature of the framework. The approach has been adopted by over 100 organizations, while the CUA, AUA, and the European Association of Urology have all made a stated effort to move toward GRADE methodology in guideline development when possible. ${ }^{4}$

When it comes to urethral strictures and reconstructive urology in general, this guideline illustrates a crux in the field - the evidence base is poor. Our clinical decisions are mostly based on comparative observational data at best, clinical principle and experience at worst; the crowned jewel of evidence-based medicine - a randomized controlled trial - is a whisper in the wind. This is where a GRADEderived guideline can punch above other, more hierarchyof-evidence-derived guideline methodologies and deliver guidance that can be more helpful in the real world. For their use of the adolopment approach, the authors should be lauded for not simply redoing what has already been done. Although perhaps not as comprehensive in terms of the scope of urethral stricture management as some might have hoped, the focus on high-impact clinical scenarios with clinically meaningful outcome measures that are clearly communicated using a transparent methodology has resulted in a real win for practicing urologists and their patients.

Competing interests: The author reports no competing personal or financial interests related to this work.

\section{References}

1. Rourke KF, Welk B, Kodama R, et al. Canadian Urological Association guideline on male urethral stricture. Can Urol Assoc J 2020;14:305-16. http://dx.doi.org/10.5489/cuai.6792.

2. Schünemann HJ, Wiercioch W, Brozek J, et al. GRADE Evidence to Decision (EtD) frameworks for adoption, adaptation, and de novo development of trustworthy recommendations: GRADE-ADOLOPMENT. I Clin Epidemiol 2017;81:101-10. https://doi.org/10.1016/i.jlinepi.2016.09.009

3. Wessells H AK, Elliott S, Gonzalez CM, et al. Male urethral stricture: American Urological Association guideline. J Urol 2017;197:182-90. https://doi.org/10.1016/i.juro.2016.07.087

4. Dahm P, Lewis J, Sultan S. Advancing Canadian Urological Association guidelines using GRADE. Can Urol Assoc J 2018;12:93-4. https://doi.org/10.5489/cuaj.5270

Correspondence: Dr. R. Christopher Doiron, Department of Urology, Queen's University, Kingston, ON, Canada; chris.doiron@queensu.ca

\section{Search guidelines by specialty, specific topic, and type.}

\section{Visit the new-and-improved CUA guidelines website and start exploring!}

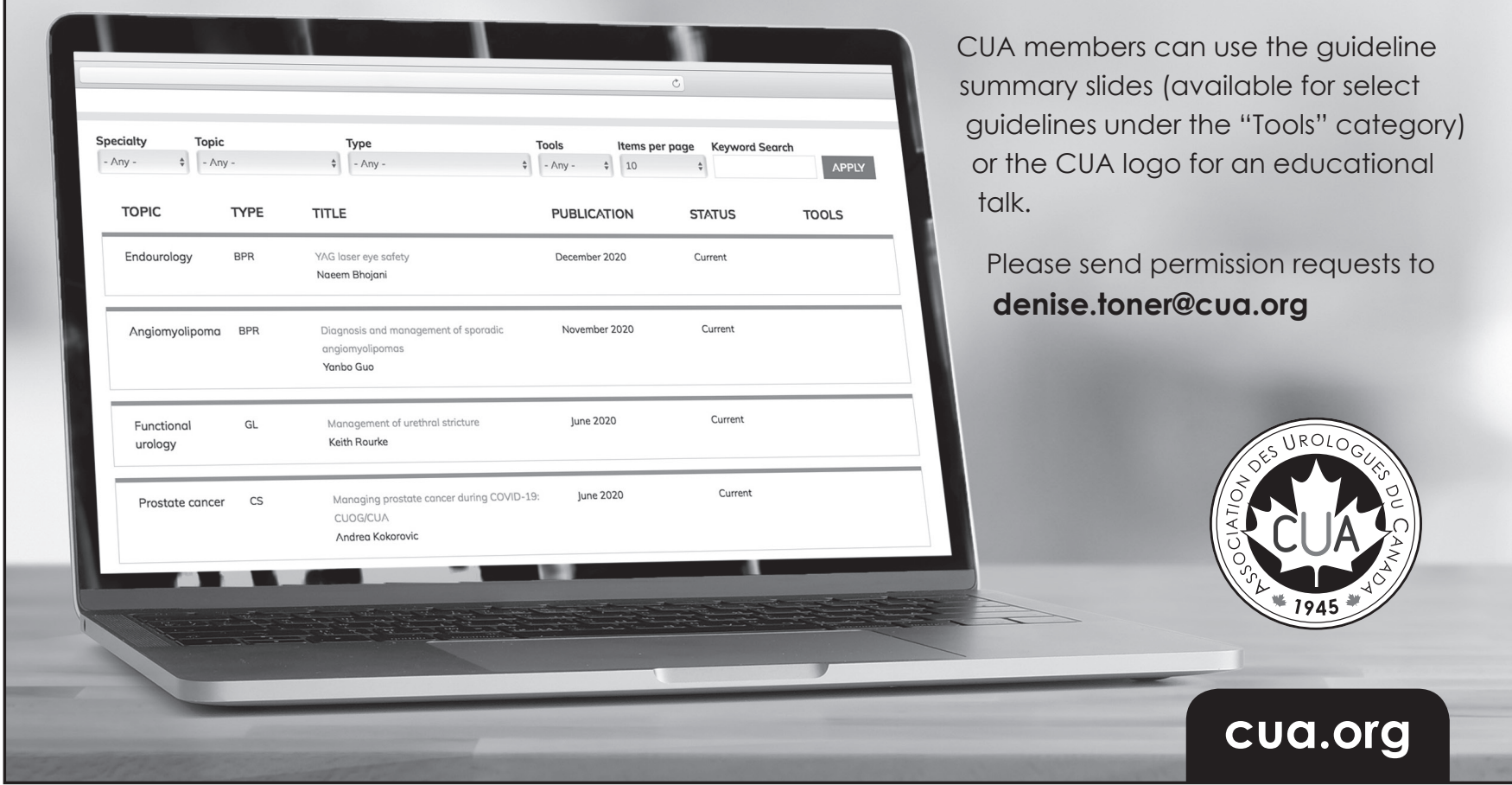

